\title{
Robust compliance topology optimization using the first-order second-moment method
}

\author{
Benedikt Kriegesmann · Julian K. Lüdeker
}

Received: date / Accepted: date

\begin{abstract}
A robust topology optimization approach is presented which uses the probabilistic first-order second-moment method for the estimation of mean value and variance of the compliance. The considered sources of uncertainty are the applied load, the spatially varying Young's modulus and the geometry with focus on the latter two. In difference to similar existing approaches for robust topology optimization, the presented approach requires only one solution of an adjoint system to determine the derivatives of the variance, which keeps the computation time close to the deterministic optimization. For validation, also the second-order fourth-moment method and Monte Carlo simulations are embedded into the optimization. For all approaches, the applicability and impact on the resulting design are demonstrated by application to benchmark examples. For random load, the firstorder second-moment approach provides unsatisfying results. For random Young's modulus and geometry however, the robust topology optimization using first-order second-moment approach provides robust designs at very little computational cost.
\end{abstract}

Keywords robust topology optimization, reliability-based topology optimization

\section{Introduction}

Deterministic design optimization can provide designs, which are very sensitive to deviation from the ideal structure or loading situation. This motivated embedding uncertainty analyses into design optimization. Elishakoff categorized different approaches to analyze

\author{
Benedikt Kriegesmann \\ Hamburg University of Technology \\ Am Schwarzenberg-Campus 4 \\ 21073 Hamburg \\ Tel.: +49 (0) 4042878 - 4857 \\ E-mail: benedikt.kriegesmann@tuhh.de \\ Julian Kajo Lüdeker \\ Hamburg University of Technology \\ Am Schwarzenberg-Campus 4 \\ 21073 Hamburg \\ Tel.: +49(0) 4042878 - 4858 \\ E-mail: julian.luedeker@tuhh.de
}


and propagate uncertainty into probabilistic methods, fuzzy set and anti-optimization with set theoretical approaches [9]. All type of methods have been used for optimization under uncertainty, as summarized for instance in the overview article by Yao et al. [35]. Though these different approaches are motivated by very different concepts, Kriegesmann et al. [20] showed for composite cylinders under axial compression that anti-optimization using the set theoretical approach and the probabilistic first-order second-moment method lead to the same response surface for optimization, when fed with the same information.

In the current paper, uncertainties are handled using probabilistic methods (for an overview see, e.g. [12,8]). Embedding probabilistic analyses in optimization is referred to as robust design optimization (RDO) or reliability-based design optimization (RBDO). RDO typically targets reducing some cost function and at the same time reducing the variance of this (or another) cost function. RBDO typically includes constraints not to exceed a certain bound with a predefined probability. RBDO is often more costly, since determining a quantile associated with a low probability often requires more computation time than determining the variance. However, it allows using sequential approaches and hence, decoupling of the optimization and the probabilistic analysis. For an overview of RDO and RBDO approaches the reader is referred to [36] and [30].

RDO and RBDO approaches have been applied to topology optimization referred to as robust topology optimization (RTO) or reliability-based topology optimization (RBTO). First works used the Solid Isotropic Material with Penalization (SIMP) approach for topology optimization of compliance and the performance measure approach for the probabilistic analysis [2,27]. Following works made use of the fact the RBTO problem can be decoupled into a deterministic optimization and subsequent probabilistic analysis, yielding a sequential approach [18, 17,25]. Luo et al. [26] followed a similar approach for probabilistic stress constraints. In the majority of these works, the applied load is considered as random input parameter. Bae et al. [2] and Kharmanda et al. [18] considered the Young's modulus as random, but constant over the design space. Hence, all random parameters considered are independent of the design. The same holds for works in which other approaches are used for the topology optimization within the RBTO, namely the level set approach $[10,7,11,33]$ and evolutionary structural optimization [19].

A Monte Carlo based RBTO approach considering random loads was presented by Zhao and Wang [37]. Papadimitriou and Mourelatos [28] presented a more efficient approach for such problems, which is based on Taylor series expansion. Jalalpour and Tootkaboni [16] also used a Taylor series based RBTO, but considered the Youngs modulus as a spatially varying parameters, described by random fields. The basis for this approach was developed for truss structures, which however was formulated in a general way and allowed its extension to topology optimization $[1,15]$.

Sigmund [31,34] suggested modeling "eroded" and "dilated" designs within the topology optimization by considering the density threshold as random. This threshold defines which density values are considered as void and solid. Schevenels et al. [29] extended this idea by considering the threshold as a spatially random variable, which allows modeling geometric deviations within the topology optimization. The RTO used Monte Carlo simulations to determine mean value and standard deviation of the compliance, resulting in large computational effort. Lazarov et al. used the same approach to model random geometry, but more efficient approaches for the probabilistic analysis, namely the (Taylor series based) perturbation technique [22] and the stochastic collocation method [21]. Though providing better efficiency than using Monte Carlo, these approaches still require significantly more computation time than standard deterministic optimization. 
The approaches based on Taylor series $[22,16,28]$ have in common, that the computational effort increases linearly with the number of random parameters for first-order approximations and quadratically for second order approximations.

In the current paper, the approach of Schevenels et al. [29] is followed to model geometric uncertainty. Furthermore, the Young's modulus is considered as a spatially random parameter, and also the load is considered as random. The probabilistic approaches used within the RTO are the first-order second-moment (FOSM) method and the second-order fourth-moment (SOFM) method. The methods are based on Taylor series of the objective function at the mean vector. The way the gradient is determined only one additional adjoint system needs to be solved per iteration. This comes at the cost that the second order approach scales with the number of design parameters and is therefore not applicable to problems of reasonable size. The focus however lies on the first-order approach. It does not require any reduction of random variables, no assumption on the type of distribution of input parameters and hence no transformation to Gaussian distribution.

The paper is structured as follows. First, the robust design optimization problem considered is given and the probabilistic approach is described, from which the gradients are derived in a general manner. Then, probabilistic approach and its gradient are given for the case of robust topology optimization for minimum compliance. Next, the first-order approach is applied to use cases, showing the potential and limitations of the approach. The results are assessed by comparison with results of second-order and Monte Carlo based optimization.

\section{Robust design optimization formulation}

Consider an probabilistic objective function $g(\mathbf{x}, \mathbf{y})$, which is a function of the random vector $\mathbf{X}$ and the set of design parameters $\mathbf{y}$. The robust design optimization problem considered is

$$
\begin{array}{r}
\min _{\mathbf{y}} f_{R D O}(\mathbf{y})=\mu_{g}(\mathbf{y})+\kappa \sigma_{g}(\mathbf{y}) \\
\text { subject to } \mathbf{c}_{n e q}(\mathbf{y}) \leq 0 \\
\mathbf{c}_{e q}(\mathbf{y})=0
\end{array}
$$

Here, $f_{R D O}$ is the objective function of the robust design optimization, $\mathbf{c}_{n e q}(\mathbf{y})$ is a vector of inequality constraints and $\mathbf{c}_{e q}(\mathbf{y})$ is a vector of equality constraint functions. The mean $\mu_{g}$ and the standard deviation $\sigma_{g}$ of the probabilistic objective function can, for instance, be approximated with Monte Carlo simulations. The scalar $\kappa$ weights the standard deviation.

In this paper probabilistic approaches are used which do not directly provide the standard deviation $\sigma_{g}$, but the variance $\sigma_{g}^{2}$. Therefore, the derivative of the optimization objective function is written as

$$
\frac{\partial f_{R D O}}{\partial y_{j}}=\frac{\partial \mu_{g}}{\partial y_{j}}+\kappa \frac{1}{2 \sqrt{\sigma_{g}^{2}}} \frac{\partial \sigma_{g}^{2}}{\partial y_{j}}
$$

\subsection{Probabilistic approach}

The first-order second-moment (FOSM) method employed here has already been proposed Cornell [6] in 1969 and has been applied widely since then. It is based on a Taylor series of the objective function expanded at the mean vector of random input parameters, and it allows approximating the mean value and variance of the objective function. The governing 
equations are given here, as they are the basis for the gradient determination of the RTO problem considered later.

FOSM shall not be mixed up with the first order reliability method (FORM) by Hasofer and Lind [14], which is based on a Taylor series expansion at the most probable point, i.e., at some limit state function. While FOSM provides only mean value and variance, the FORM approach allows determining probability of exceedance (i.e. a probability of failure) and in general requires solving an optimization problem. Consider a objective function $g(\mathbf{x})$, which is a function of the random vector $\mathbf{x}$ with the probability density function $f_{\mathbf{X}}(\mathbf{x})$. The mean value $\mu_{g}$ and variance $\sigma_{g}^{2}$ of the objective function are given by

$$
\mu_{g}=\int_{-\infty}^{\infty} g(\mathbf{x}) f_{\mathbf{X}}(\mathbf{x}) d \mathbf{x}
$$

and

$$
\sigma_{g}^{2}=\int_{-\infty}^{\infty}\left(g(\mathbf{x})-\mu_{g}\right)^{2} f_{\mathbf{X}}(\mathbf{x}) d \mathbf{x}
$$

The Taylor series of $g$ expanded at the mean vector of random input parameters $\boldsymbol{\mu}$ reads

$$
g(\mathbf{x})=g(\boldsymbol{\mu})+\sum_{i=1}^{n} \frac{\partial g(\boldsymbol{\mu})}{\partial x_{i}}\left(x_{i}-\mu_{i}\right)+\frac{1}{2} \sum_{i=1}^{n} \sum_{j=1}^{n} \frac{\partial^{2} g(\boldsymbol{\mu})}{\partial x_{i} \partial x_{j}}\left(x_{i}-\mu_{i}\right)\left(x_{j}-\mu_{j}\right)+\ldots
$$

Inserting a second-order Taylor series of $g$ into eq. (3) yields

$$
\mu_{g} \approx g(\boldsymbol{\mu})+\frac{1}{2} \sum_{i=1}^{n} \sum_{j=1}^{n} \frac{\partial^{2} g(\boldsymbol{\mu})}{\partial x_{i} \partial x_{j}} \operatorname{cov}\left(X_{i}, X_{j}\right)
$$

Any random vector $\mathbf{X}$ can be transformed to a vector with uncorrelated entries $\mathbf{Z}$ (see section A.1). Then, the approximation of the mean reads

$$
\mu_{g} \approx g(\boldsymbol{\mu})+\frac{1}{2} \sum_{i=1}^{n} \frac{\partial^{2} g(\boldsymbol{\mu})}{\partial z_{i}^{2}} \operatorname{var}\left(Z_{i}\right)
$$

In the same manner, the variance can be approximated. For an uncorrelated random vector $\mathbf{Z}$, the second order variance approximation is given by

$$
\begin{aligned}
\sigma_{g}^{2} \approx g_{\boldsymbol{\mu}}^{2}+ & \sum_{i=1}^{n}\left(\frac{\partial g}{\partial z_{i}}\right)^{2} \mu_{i, 2}+\frac{1}{4} \sum_{i=1}^{n}\left(\frac{\partial^{2} g}{\partial z_{i}^{2}}\right)^{2} \mu_{i, 4}+g_{\mu} \sum_{i=1}^{n} \frac{\partial^{2} g}{\partial z_{i}^{2}} \mu_{i, 2}+\sum_{i=1}^{n} \frac{\partial g}{\partial z_{i}} \frac{\partial^{2} g}{\partial z_{i}^{2}} \mu_{i, 3} \\
& +\frac{1}{2} \sum_{i=1}^{n} \sum_{j=i+1}^{n} \frac{\partial^{2} g}{\partial z_{i}^{2}} \frac{\partial^{2} g}{\partial z_{j}^{2}} \mu_{i, 2} \mu_{j, 2}+\sum_{i=1}^{n} \sum_{j=i+1}^{n}\left(\frac{\partial^{2} g}{\partial z_{i} \partial z_{j}}\right)^{2} \mu_{i, 2} \mu_{j, 2}-\mu_{g}^{2}
\end{aligned}
$$

where $g_{\boldsymbol{\mu}}=g(\boldsymbol{\mu})$ and

$$
\mu_{i, k}=\int_{-\infty}^{\infty}\left(z_{i}-\mu_{i}\right)^{k} f_{X}\left(z_{i}\right) d z_{i}
$$

Using a first-order Taylor series yields the following approximations for mean value and variance of $g$.

$$
\begin{aligned}
\mu_{g} & \approx g(\boldsymbol{\mu}) \\
\sigma_{g}^{2} & \approx \sum_{i=1}^{n} \sum_{j=1}^{n} \frac{\partial g}{\partial x_{i}} \frac{\partial g}{\partial x_{j}} \operatorname{cov}\left(X_{i}, X_{j}\right)=\sum_{i=1}^{n}\left(\frac{\partial g}{\partial z_{i}}\right)^{2} \mu_{i, 2}
\end{aligned}
$$


Here, only second-order moments of the input variables are required. Therefore, the approach is referred to as first-order second-moment (FOSM) method [12]. For the secondorder approach, fourth-order moments of the input parameters are required and hence, the approach will be referred to as second-order fourth-moment (SOFM) method.

For FOSM it is not mandatory to transform the parameters to uncorrelated parameters, but for SOFM it is. The transformation and how it has to be treated for the differentiation with respect to design variables is given and discussed in Appendix A.1.

\subsection{Derivatives of approximated mean and variance}

For solving the robust design optimization problem given in eq. (1) with gradient based methods, the derivatives of the mean $\mu_{g}$ and the variance $\sigma_{g}^{2}$ with respect to the design variables $y_{k}$. For the SOFM approach, the derivatives are given by

$$
\frac{\partial \mu_{g}}{\partial y_{k}} \approx \frac{\partial g}{\partial y_{k}}+\frac{1}{2} \sum_{i=1}^{n} \frac{\partial^{3} g}{\partial z_{i}^{2} \partial y_{k}} \mu_{i, 2}
$$

and

$$
\begin{array}{r}
\frac{\partial \sigma_{g}^{2}}{\partial y_{k}}=2 g_{\boldsymbol{\mu}} \frac{\partial g}{\partial y_{k}}+2 \sum_{i=1}^{n} \frac{\partial g}{\partial z_{i}} \frac{\partial^{2} g}{\partial z_{i} \partial y_{k}} \mu_{i, 2}+\frac{1}{2} \sum_{i=1}^{n} \frac{\partial^{2} g}{\partial z_{i}^{2}} \frac{\partial^{3} g}{\partial z_{i}^{2} \partial y_{k}} \mu_{i, 4}+\frac{\partial g}{\partial y_{k}} \sum_{i=1}^{n} \frac{\partial^{2} g}{\partial z_{i}^{2}} \mu_{i, 2} \\
+\sum_{i=1}^{n} \frac{\partial^{2} g}{\partial z_{i} \partial y_{k}} \frac{\partial^{2} g}{\partial z_{i}^{2}} \mu_{i, 3}+\frac{1}{2} \sum_{i=1}^{n} \sum_{j=i+1}^{n}\left(\frac{\partial^{3} g}{\partial z_{i}^{2} \partial y_{k}} \frac{\partial^{2} g}{\partial z_{j}^{2}}+\frac{\partial^{2} g}{\partial z_{i}^{2}} \frac{\partial^{3} g}{\partial z_{j}^{2} \partial y_{k}}\right) \mu_{i, 2} \mu_{j, 2} \\
+2 \sum_{i=1}^{n} \sum_{j=i+1}^{n} \frac{\partial^{2} g}{\partial z_{i} \partial z_{j}} \frac{\partial^{3} g}{\partial z_{i} \partial z_{j} \partial y_{k}} \mu_{i, 2} \mu_{j, 2}-2 \mu_{g} \frac{\partial \mu_{g}}{\partial y_{k}}
\end{array}
$$

Hence, the derivatives of the objective function, which are required for solving an RDO problem with the second-order approximation, are

$$
\frac{\partial g}{\partial z_{i}}, \frac{\partial g}{\partial y_{k}}, \frac{\partial^{2} g}{\partial z_{i} \partial y_{k}}, \frac{\partial^{2} g}{\partial z_{i} \partial z_{j}}, \frac{\partial^{3} g}{\partial z_{i} \partial z_{j} \partial y_{k}}
$$

For the FOSM method, the derivatives are given by

$$
\frac{\partial \mu_{g}}{\partial y_{k}} \approx \frac{\partial g}{\partial y_{k}}
$$

and

$$
\frac{\partial \sigma_{g}^{2}}{\partial y_{k}}=2 \sum_{i=1}^{n} \frac{\partial g}{\partial z_{i}} \frac{\partial^{2} g}{\partial z_{i} \partial y_{k}} \mu_{i, 2}
$$

Hence, for the FOSM method only the first three terms of eq. (13) are required. 


\section{Robust topology optimization for minimum compliance}

In the following sections, the probabilistic objective function considered is the compliance $c$ of a structure. The objective of the robust design optimization is to minimize the mean value $\mu_{c}$ and the standard deviation $\sigma_{c}$ of the compliance for a given volume ratio $v$. Each design variable $\rho_{e}$ is associated to one finite element of the design space. The stiffnesses of the finite elements vary dependent on the design variables $\rho_{e}$ according to the Simplified Isotropic Material with Penalization (SIMP) approach [3] (see eq. (50)). The design variables are summarized in the design vector $\rho$. The optimization problem hence reads

$$
\begin{aligned}
& \min _{\boldsymbol{\rho}} \mu_{c}(\boldsymbol{\rho})+\kappa \sigma_{c}(\boldsymbol{\rho}) \\
& \text { subject to } \\
& \frac{V(\boldsymbol{\rho})}{V_{0}} \leq v \\
& 0<\rho_{\min } \leq \rho_{e} \leq 1 \\
& \mathbf{K u}=\mathbf{f}
\end{aligned}
$$

The compliance $c=\mathbf{u}^{T} \mathbf{f}$ is determined from the load vector $\mathbf{f}$ and the displacement vector $\mathbf{u}$, which is obtained from a linear finite element (FE) analysis. $\mathbf{K}$ is the stiffness matrix of the FE model.

In the following subsections, the gradient of variance is determined for the case of random Young's modulus and geometry only. As will be shown in section 4.1, FOSM does not work well for random loads. It is furthermore worth mentioning that for linear elastic problems, Monte Carlo simulations can be carried out extremely cheap by running unit load cases and superposing them.

\subsection{Filtering design variables and modeling random geometry}

The design variables $\rho_{e}$ are filtered and projected using the same approach as in [29]. The filter function and its derivative equal

$$
\tilde{\rho}_{e}=\frac{\sum_{i=1}^{Q} w_{e i} v_{i} \rho_{i}}{\sum_{i=1}^{Q} w_{e i} v_{i}} \text { and } \frac{\partial \tilde{\rho}_{e}}{\partial \rho_{k}}=\frac{w_{e k} v_{k}}{\sum_{i=1}^{Q} w_{e i} v_{i}} \text { with } w_{e i}=\max \left(0, R-r_{e i}\right)
$$

Here, $v_{i}$ is the volume of the $i$-th element, $r_{e i}$ is the distance of the $i$-th element to the $e$ th element and $R$ is the filter radius. $Q$ can be the number of elements, but in practical implementation it is the number of neighbor elements.

As discussed by Clausen and Andreassen [5], by using the filter function (17) it is implicitly assumed that the design variables are continuous at the edge of the design space. To avoid this effect the filter needs to be adjusted near the design space edge. The sum in the denominator is extended to the outside of the design space, assuming that there is zero density. This modification is however not applied to areas of boundary conditions and load application. The approach is referred to as padding in the following. An example of design variables $\rho$ and their associated filtered variables $\tilde{\rho}$ without and with padding are shown in figure 1, 2 and 3 . In this example, the boundary conditions are at the top of the design space. 


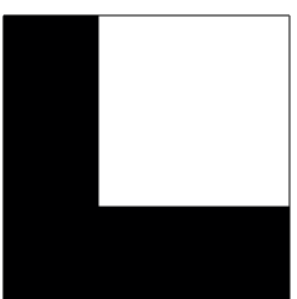

Fig. 1 Example field of design variables $\rho$

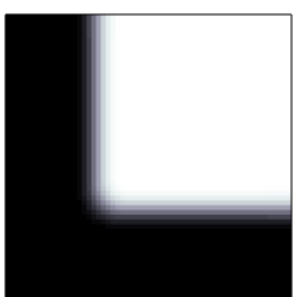

Fig. 2 Example field of filtered variables $\tilde{\rho}$ without padding

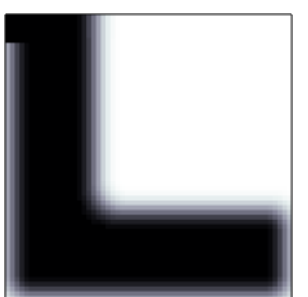

Fig. 3 Example field of filtered variables $\tilde{\rho}$ with padding

Therefore, there is a small region on the top left corner of figure 3, where the padding is not applied, even if it is applied to the rest of the structure.

The filtered variables $\tilde{\rho}_{e}$ are projected to $\overline{\tilde{\rho}}_{e}$ using the Heaviside approximation (43). These projected variables $\overline{\tilde{\rho}}_{e}$ are used to scale the stiffness of the finite elements. Examples for projected variables $\overline{\tilde{\rho}}$ without and with padding and using a constant projection threshold $\eta$ are shown in figure 4 and 5 .

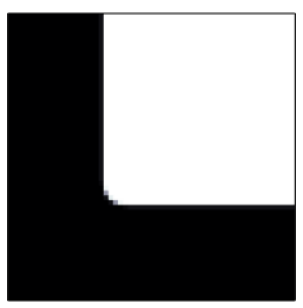

Fig. 4 Example field of projected variables $\overline{\tilde{\rho}}$ without padding using a fixed $\eta$

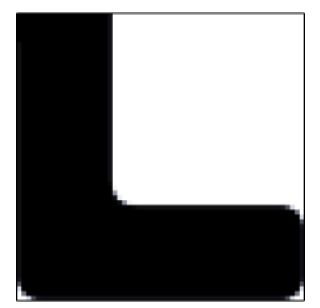

Fig. 5 Example field of projected variables $\overline{\tilde{\rho}}$ with padding using a fixed $\eta$

Schevenels et al. [29] suggested to consider the threshold $\eta$ as a spatially varying variable to model randomness of geometry. An example for a random field of $\eta$ is shown in figure 6 . The projected variables $\overline{\tilde{\rho}}$ obtained with this varying $\eta$ are shown in figure 7 and 8 without and with padding. The example shows how important the consideration of filter boundary conditions (padding) according to Clausen and Andreassen [5] is especially for modeling random geometric variations at the design space edge.

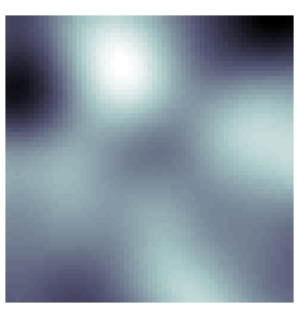

Fig. 6 Example random field of the projection threshold $\eta$

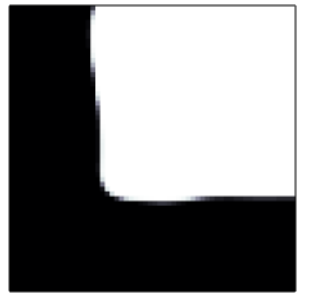

Fig. 7 Example field of projected variables $\overline{\tilde{\rho}}$ without padding using a variable $\eta$

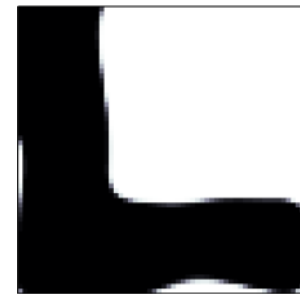

Fig. 8 Example field of projected variables $\overline{\tilde{\rho}}$ with padding using a variable $\eta$ 


\subsection{Gradient determination}

In this section, the gradient of mean value and variance estimated by the first-order secondmoment (FOSM) approach are derived. A spatially varying random variable $\alpha$ is considered, which influences the stiffness matrix, but not the load. Hence, $\alpha$ represents the Young's modulus $E$ or the projection threshold $\eta$.

Using the FOSM approximation (10), the mean value and the variance of the compliance, as well as the derivative of the mean value, are determined from the displacement vector $\mathbf{u}$ and derivatives of $\mathbf{K}$.

$$
\begin{gathered}
\mu_{c} \approx c \\
\frac{\partial \mu_{c}}{\partial \overline{\tilde{\rho}}_{k}}=\frac{\partial c}{\partial \overline{\tilde{\rho}}_{k}}=-\mathbf{u}^{T} \frac{\partial \mathbf{K}}{\partial \overline{\tilde{\rho}}_{e}} \mathbf{u} \\
\sigma_{c}^{2} \approx \sum_{i=1}^{n} \sum_{j=1}^{n}\left(\mathbf{u}^{T} \frac{\partial \mathbf{K}}{\partial \alpha_{i}} \mathbf{u}\right)\left(\mathbf{u}^{T} \frac{\partial \mathbf{K}}{\partial \alpha_{j}} \mathbf{u}\right) \operatorname{cov}\left(\alpha_{i}, \alpha_{j}\right)
\end{gathered}
$$

Since the derivatives of $\mathbf{K}$ are given explicitly, only the equilibrium condition $\mathbf{K u}=\mathbf{f}$ has to be solved. For the derivative of the variance the term $\lambda^{T}(\mathbf{K} \mathbf{u}-\mathbf{f})$ is added to eq. (20). The derivative then reads

$$
\begin{array}{r}
\frac{\partial \sigma_{c}^{2}}{\partial \tilde{\tilde{\rho}}_{k}}=\sum_{i=1}^{n} \sum_{j=1}^{n}\left(2 \mathbf{u}^{T} \frac{\partial \mathbf{K}}{\partial \alpha_{i}} \frac{\partial \mathbf{u}}{\partial \overline{\tilde{\rho}}_{k}}+\mathbf{u}^{T} \frac{\partial^{2} \mathbf{K}}{\partial \alpha_{i} \partial \overline{\tilde{\rho}}_{k}} \mathbf{u}\right)\left(\mathbf{u}^{T} \frac{\partial \mathbf{K}}{\partial \alpha_{j}} \mathbf{u}\right) \\
+\left(\mathbf{u}^{T} \frac{\partial \mathbf{K}}{\partial \alpha_{i}} \mathbf{u}\right)\left(2 \mathbf{u}^{T} \frac{\partial \mathbf{K}}{\partial \alpha_{j}} \frac{\partial \mathbf{u}}{\partial \overline{\tilde{\rho}}_{k}}+\mathbf{u}^{T} \frac{\partial^{2} \mathbf{K}}{\partial \alpha_{j} \partial \tilde{\tilde{\rho}}_{k}} \mathbf{u}\right) \operatorname{cov}\left(\alpha_{i}, \alpha_{j}\right)+\boldsymbol{\lambda}^{T}\left(\frac{\partial \mathbf{K}}{\partial \overline{\tilde{\rho}}_{k}} \mathbf{u}+\mathbf{K} \frac{\partial \mathbf{u}}{\partial \overline{\tilde{\rho}}_{k}}\right)
\end{array}
$$

Simplifying the expression and isolating $\frac{\partial \mathbf{u}}{\partial \tilde{\rho}_{k}}$ yields

$$
\begin{aligned}
& \frac{\partial \sigma_{c}^{2}}{\partial \tilde{\rho}_{k}}=2\left(\mathbf{u}^{T} \frac{\partial^{2} \mathbf{K}}{\partial \alpha_{k} \partial \tilde{\tilde{\rho}}_{k}} \mathbf{u}\right) \sum_{j=1}^{n}\left(\mathbf{u}^{T} \frac{\partial \mathbf{K}}{\partial \alpha_{j}} \mathbf{u}\right) \operatorname{cov}\left(\alpha_{k}, \alpha_{j}\right)+\lambda^{T} \frac{\partial \mathbf{K}}{\partial \tilde{\tilde{\rho}}_{k}} \mathbf{u} \\
& +\left\{\left[4 \sum_{i=1}^{n} \sum_{j=1}^{n} \operatorname{cov}\left(\alpha_{i}, \alpha_{j}\right)\left(\mathbf{u}^{T} \frac{\partial \mathbf{K}}{\partial \alpha_{j}} \mathbf{u}\right)\left(\mathbf{u}^{T} \frac{\partial \mathbf{K}}{\partial \alpha_{i}}\right)\right]+\lambda^{T} \mathbf{K}\right\} \frac{\partial \mathbf{u}}{\partial \tilde{\tilde{\rho}}_{k}}
\end{aligned}
$$

Requesting the term in curly brackets to equal zero yields the following system of linear equations.

$$
\mathbf{K} \boldsymbol{\lambda}=-4 \sum_{i=1}^{n} \sum_{j=1}^{n}\left(\mathbf{u}^{T} \frac{\partial \mathbf{K}}{\partial \alpha_{j}} \mathbf{u}\right) \operatorname{cov}\left(\alpha_{i}, \alpha_{j}\right)\left(\frac{\partial \mathbf{K}}{\partial \alpha_{i}} \mathbf{u}\right)
$$

Having solved this equation for $\boldsymbol{\lambda}$, the derivative is given by

$$
\frac{\partial \sigma_{c}^{2}}{\partial \tilde{\tilde{\rho}}_{k}}=2\left(\mathbf{u}^{T} \frac{\partial^{2} \mathbf{K}}{\partial \alpha_{k} \partial \tilde{\tilde{\rho}}_{k}} \mathbf{u}\right) \sum_{j=1}^{n}\left(\mathbf{u}^{T} \frac{\partial \mathbf{K}}{\partial \alpha_{j}} \mathbf{u}\right) \operatorname{cov}\left(\alpha_{k}, \alpha_{j}\right)+\lambda^{T} \frac{\partial \mathbf{K}}{\partial \tilde{\tilde{\rho}}_{k}} \mathbf{u}
$$

Compared to the deterministic optimization, one additional solution of a system of linear equations is required, namely eq. (23), for which the already decomposed stiffness matrix is used.

When $\alpha$ is considered to be the spatially varying Young's modulus, the required derivatives of the stiffness matrix

$$
\frac{\partial \mathbf{K}}{\partial \alpha_{i}}=\frac{\partial \mathbf{K}}{\partial E_{i}}
$$


and

$$
\frac{\partial^{2} \mathbf{K}}{\partial \alpha_{i} \partial \tilde{\tilde{\rho}}_{i}}=\frac{\partial^{2} \mathbf{K}}{\partial E_{i} \partial \tilde{\tilde{\rho}}_{i}}
$$

are given by eq. (71) and eq. (72). When considering random geometry, represented by a spatially varying projection threshold, the derivatives of the stiffness matrix

$$
\frac{\partial \mathbf{K}}{\partial \alpha_{i}}=\frac{\partial \mathbf{K}}{\partial \eta_{i}}=\frac{\partial \mathbf{K}}{\partial \tilde{\bar{\rho}}_{i}} \frac{\partial \overline{\tilde{\rho}}_{i}}{\partial \eta_{i}}
$$

and

$$
\frac{\partial^{2} \mathbf{K}}{\partial \alpha_{i} \partial \overline{\tilde{\rho}}_{i}}=\frac{\partial^{2} \mathbf{K}}{\partial \eta_{i} \partial \overline{\tilde{\rho}}_{i}}=\frac{\partial^{2} \mathbf{K}}{\partial \overline{\tilde{\rho}}_{i} \partial \overline{\tilde{\rho}}_{i}} \frac{\partial \overline{\tilde{\rho}}_{i}}{\partial \eta_{i}}
$$

are given by eq. (80) and eq. (82).

When using the SOFM approach, mixed second partial derivatives of $\mathbf{u}$ with respect to $\overline{\tilde{\rho}}$ and a random variable, of with respect to a different $\overline{\tilde{\rho}}$ occur. The authors did not see any possibility to isolate theses expressions and using the adjoint method for determining the gradient. Hence, in the following section the direct differentiation is used when using the SOFM method, by simply inserting the expressions given in appendix A.3 into eq. (11) and (12). The computational cost of this approach does not allow any practical application and is only used for comparison.

Table 1 Comparison of computational cost for determining the gradient of the objective function of the RTO problem considered using different probabilistic approaches

\begin{tabular}{lll}
\hline \hline Probabilistic approach & Solutions of Ku=f & Solutions of adjoint systems \\
\hline FOSM (adjoint of variance) & 1 & 1 \\
SOFM (direct differentiation) & 1 & $N_{\rho}+N_{\alpha}+N_{\rho} \times N_{\alpha}$ \\
Perturbation approach (first order) & 1 & $N_{\alpha}$ \\
Perturbation approach (second order) & 1 & $N_{\alpha}+N_{\alpha}^{2}$ \\
Monte Carlo & $N_{\text {samp }}$ & 0 \\
\hline \hline
\end{tabular}

$N_{\rho}$ : number of design variables

$N_{\alpha}$ : number of random variables

$N_{\text {samp }}$ : number of samples/realizations used

In [22], Lazarov et al. present a RTO approach using the perturbation technique, which is also based on Taylor series expansions. While in the current paper the Taylor series is directly applied to approximate the objective function (namely the compliance), Lazarov et al. use Taylor expansions for the stiffness matrix $\mathbf{K}$, the displacement $\mathbf{u}$ and the force f. These expansions are used to obtain required derivatives, where the number of adjoint systems to be solved only depends on the number of random parameters. An overview and comparison of the computational efforts is given in table 1 .

3.3 Connection with reliability based topology optimization with compliance constraint

The FOSM approach and hence, the gradients derived above can also be used for reliability based topology optimization as, for instance, also carried out by Jalalpour and Tootkaboni 
[16]. To depict this, consider the deterministic optimization problem, where now compared to (16) objective function and constraint are interchanged.

$$
\begin{aligned}
& \underset{\boldsymbol{\rho}}{\min } V(\boldsymbol{\rho}) \\
& \text { subject to } \\
& c(\boldsymbol{\rho}) \leq c_{0} \\
& 0<\rho_{\min } \leq \rho_{e} \leq 1 \\
& \mathbf{K u}=\mathbf{f}
\end{aligned}
$$

Here, $c_{0}$ is the maximum allowed compliance. In the corresponding RBTO problem, $c_{0}$ may be exceed with a given probability $\bar{p}$. The optimization problem then reads

$$
\begin{aligned}
& \min _{\boldsymbol{\rho}} V(\boldsymbol{\rho}) \\
& \text { subject to } \\
& P\left(C(\boldsymbol{\rho})>c_{0}\right)=1-F_{C}\left(c_{0}\right) \leq \bar{p} \\
& 0<\rho_{\min } \leq \rho_{e} \leq 1 \\
& \mathbf{K} \mathbf{u}=\mathbf{f}
\end{aligned}
$$

The FOSM and SOFM approaches only provide stochastic moments but no probability of exceedance $F_{C}\left(c_{0}\right)$ (in difference to approximations at the most probable point [14]). Only, when a certain type of distribution is assumed for $C, F_{C}$ is given based on the moments. Any distribution can be normalized such that its mean equals 0 and its variance equals 1 . Then, $F_{C}$ can be expressed in terms of the normalized distribution $F_{N}$.

$$
F_{C}\left(c_{0}\right)=F_{N}\left(\frac{c_{0}-\mu_{c}}{\sigma_{c}}\right)
$$

Then, the probabilistic constraint of (30) can be expressed as

$$
\begin{aligned}
& P\left(C(\rho)>c_{0}\right)=1-F_{N}\left(\frac{c_{0}-\mu_{c}(\rho)}{\sigma_{c}(\rho)}\right) \leq \bar{p} \\
& \Leftrightarrow \frac{c_{0}-\mu_{c}(\rho)}{\sigma_{c}(\rho)} \geq F_{N}^{-1}(1-\bar{p})=\bar{b} \\
& \Leftrightarrow 0 \geq \mu_{c}(\rho)+\bar{b} \sigma_{c}(\rho)-c_{0}
\end{aligned}
$$

The value $\bar{b}$ is dependent on the chosen distribution type and the desired probability $\bar{p}$. (In the current formulation $\bar{b}$ equals the reliability index, typically denoted as $\beta$, see, e.g., [12].) For instance, assuming Gauss distribution and choosing probability of $\bar{p}=1 \%$ yields $\bar{b}=2.33$. For the same $\bar{p}$, assuming Gumbel distribution of $C$ yields $\bar{b}=1.64$ and assuming Logistic distribution yields $\bar{b}=4.60$. It is however difficult to justify a distribution type in advance. (For the Monte Carlo simulations in the following sections the compliance turned out to be highly skewed.) The intention of this section however is mainly to motivate a value for $\kappa$ in eq. (16). As can be seen form eq. (32), the objective function (16) can be seen as an upper bound, associated with a certain associated with a certain probability of exceedance if $\kappa$ is replaced by $\bar{b}$. This helps to choose an order of magnitude of $\kappa$. 


\section{Example and results}

The presented approaches are applied to two examples from literature. For all examples, the method of moving asymptotes [32] with some modifications suggested by Li and Khandelwal [24]. The initial move limit, relaxation factor and tightening factor were chosen conservatively with $s_{i n i}=0.2, s_{i n c r}=1.2$ and $s_{\text {decr }}=0.7$. All examples are run with a penalty factor of $p=3$, a Young's modulus of $E=1$ and a Poisson's ratio of $v=0.3$. For the Heaviside approximation (43), the value of $\eta=0.5$ was kept constant through the optimization (except for modeling geometric imperfections, where $\eta$ varies). The slope parameter $\beta$ was increased gradually, starting with $\beta=1$ and increasing it every iteration by 0.25 up to a maximum value of $\beta=10$, unless otherwise stated. A further increase of $\beta$ in many cases jeopardize the convergence.

Note that for values of $\beta$ close to 1 , the projection function (43) is $\overline{\tilde{\rho}} \approx \tilde{\rho}$, which is equivalent to not performing the projection step. Since the geometric variation come into play through the projection, this means that for the case of random geometry the first iterations are performed as for the deterministic optimization and that the influence of randomness increases continuously.

\subsection{RTO of tension bar with random load}

The methods derived above are applied to an example with random load. The example is shown in figure 9, where the horizontal force is random with zero mean. Similar (or the same) example has been considered in many works, in which the load is considered as random variable $[27,10,26,4]$. All relevant parameters used within this example are given in table 2. The optimization problem (16) is solved with $\kappa=3$.

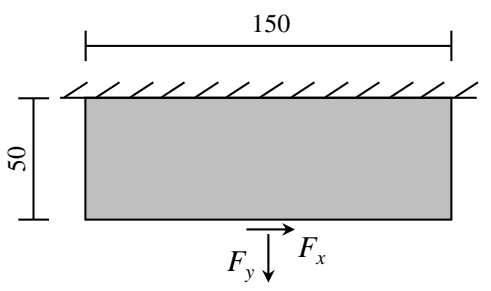

Fig. 9 Design space and load of the tension bar example

Table 2 Properties of the tension plate, similar to [26]

\begin{tabular}{ll}
\hline \hline Dimensions: & $150 \times 50$ \\
Element size & $L_{e}=1$ \\
Volume fraction & $V / V_{0}=10 \%$ \\
Filter radius & $r=4$ \\
Deterministic load & $F_{y}=1$ \\
Random load & $F_{x}: \mu=0, \sigma=1 / 3$ \\
\hline \hline
\end{tabular}




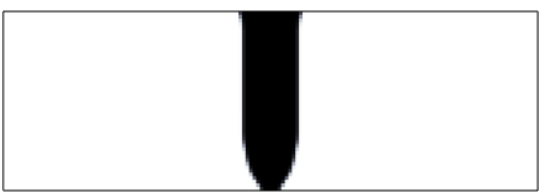

Fig. 10 Result of deterministic topology optimization of the tension bar

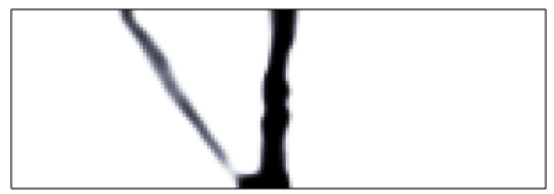

Fig. 12 Result of robust topology optimization with random load orientation using FOSM

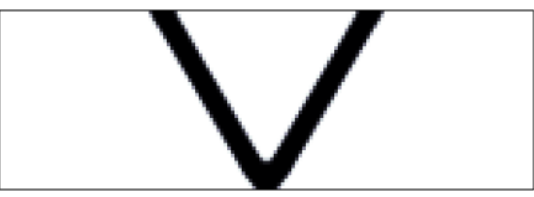

Fig. 11 Result of robust topology optimization with random load orientation using Monte Carlo

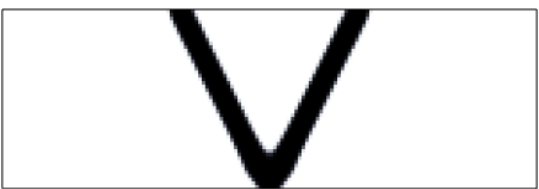

Fig. 13 Result of robust topology optimization with random load orientation using SOFM

The deterministic optimization provides a straight bar (see figure 10), since the horizontal force is not present in this optimization. The RTO using Monte Carlo simulation provides a two bar design (figure 11), as it is reported in literature. Also result from the RTO using the SOFM approach shown in figure 13 provides a two bar design, but with more inclination compared to the optimization with Monte Carlo. The RTO using FOSM fails for this example (see figure 12). The Monte Carlo simulations have been carried out with 100 samples. The realization have not been modified during the optimization to ensure smooth convergence.

In table 3, the mean value $\mu_{c}$ and standard deviation $\sigma_{c}$ of the compliance of the final result is given for all approaches. Both moments were determined by Monte Carlo simulation with 100 realizations. For FOSM and SOFM, the moments determined by the approaches themselves are given in addition. The large discrepancy of $\mu_{c}$ and $\sigma_{c}$ given by FOSM and Monte Carlo shows how inaccurate the assumption of a linear objective function is for the given case. For SOFM, the results are in much better agreement.

The deterministic optimization requires one solution of the equilibrium system per iteration. Optimizing with Monte Carlo here requires 100 times as much. For FOSM, only one adjoint system needs to be solved additionally compared to the deterministic approach, but it provides very unsatisfying results for the given example. For the optimization with SOFM $n+m+n \times m=5000+2+2 \times 5000=15002$ adjoint systems need to be solved (see section 3 ). Hence, the computational effort is much larger than for the Monte Carlo, even for the small example considered.

Table 3 Mean value and standard deviation of the compliance of the design obtained by different RTO approaches

\begin{tabular}{lll}
\hline \hline Approach & $\mu_{c}$ & $\sigma_{c}$ \\
\hline Deterministic & 23.3 & 33.9 \\
Monte Carlo & 11.4 & 4.4 \\
FOSM & $14.0^{*}, 35.3$ & $0.5^{*}, 39.7$ \\
SOFM & $10.0^{* *}, 10.5$ & $3.2^{* *}, 5.0$ \\
\hline \hline
\end{tabular}

*determined by FOSM, **determined by SOFM 
Figure 14 shows that the deterministically optimized design has a lower compliance for $F_{x}=0$, which is at the cost of a much higher increase of the compliance for $F_{x} \neq 0$.

What can also be seen from figure 14 is why the FOSM approach fails to provide a robust design. The FOSM approach implies the assumption that the objective function (here the compliance) is a linear function of the random variable (here, the load $F_{x}$ ). Since the second derivative of the compliance with respect to loads is constant (see eq. (61)), the function is in fact quadratic. For $F_{x}=0$ the compliance has a minimum and hence, the first derivative equals zero (see figure 14). Since only the first derivatives are considered for the first-order variance approximation (10), the variance is estimated to equal zero. Hence, RTO with FOSM provides the same result as a deterministic optimization for the considered case. The same phenomenon is observed when the mean value of $F_{x}$ is non-zero, because also then the compliance has a minimum with respect to $F_{x}$ at the mean of $F_{x}$. This phenomenon is

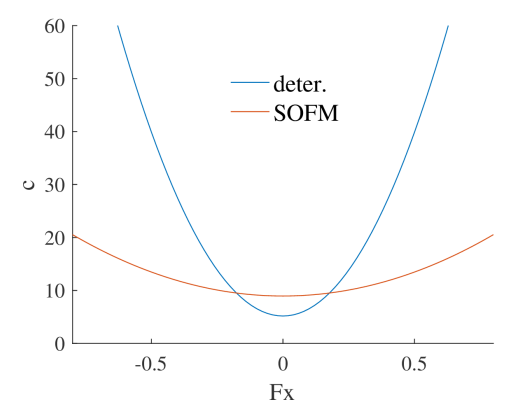

Fig. 14 Compliance as a function of the horizontal load $F_{x}$ for the deterministically optimized structure and the optimal design provided by the SOFM approach

well known and already reported in textbooks [13] and therefore, first-order approaches are not recommended for robust design optimization. However, the occurrence of this effect depends on the random parameter considered. For instance, the compliance is a monotonically increasing function of Young's modulus and therefore does not have a minimum at the mean. This is considered in the following subsection.

For the example considered in this section, the presence of random geometry or random Young's modulus provides the same optimal design as the deterministic optimization. Therefore, different examples are considered in the following sections.

\subsection{RTO of L-beam with random geometry or random Young's modulus}

In this section, robust topology optimization of the L-beam shown in figure 15 is carried out. Similar examples have been considered for RTO and RBTO approaches in [11] and [26]. The properties of the example considered are summarized in table 4 . The result of the deterministic optimization is shown in figure 16. For the RTO, the optimization problem (16) is now solved with $\kappa=5$ in order to enforce the influence of the variance.

First, the geometry is considered as random, which is modeled by spatial variation of the projection threshold $\eta$ and which is assumed to be uniformly distributed in the interval $[0.3,0.7]$. For the FOSM approach, the variance of the distribution are required, which are given in eq. (33) for uniform distribution of the interval $\left[\eta^{-}, \eta^{+}\right]$.

$$
\sigma_{\eta}^{2}=\left(\eta^{+}-\eta^{-}\right)^{2} / 12
$$




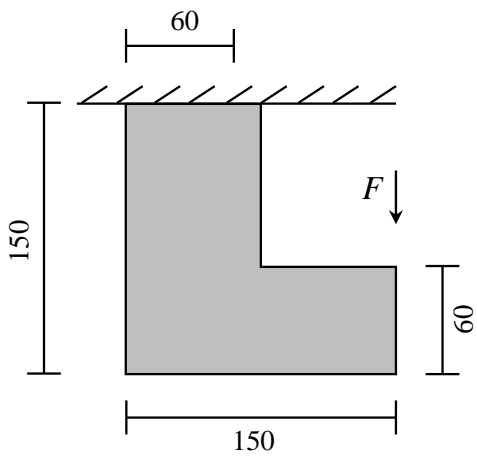

Fig. 15 Design space and load of the L-beam example

Table 4 Properties of L-beam example

\begin{tabular}{ll}
\hline \hline Dimensions: & $150 \times 150$ \\
Element size & $L_{e}=1$ \\
Volume fraction & $V / V_{0}=20 \%$ \\
Filter radius & $r=3$ \\
Applied load & $F=1$ \\
Correlation lengths & $l_{c}=20$ \\
\hline \hline
\end{tabular}

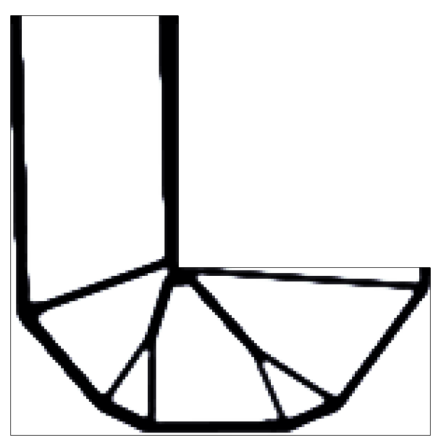

Fig. 16 Result of deterministic topology optimization of the L-beam

For the Monte Carlo simulations, realization of a Gaussian random field $\xi$ are generated, which are transformed to realizations of the uniformly distributed threshold $\eta$ via their cumulative distribution functions $F_{\xi}$ and $F_{\eta}$ as follows (see also [29]).

$$
\eta=F_{\eta}^{-1}\left(F_{\xi}(\xi)\right)
$$

The results from optimizations with Monte Carlo simulations and with FOSM method are shown in figure 17 and 18, respectively. Both designs have slightly more material at the inner corner compared to the deterministic results (see figure 19 and 20). The reason is that variations of the geometry at the corner penalize the stiffness of the design. Besides that, the topology of all three designs is very similar. The locations of the connecting bars obtained with Monte Carlo differ slightly and turned out to be slightly different in each optimization run, depending on the realizations of the random field. The results in table 5 show 
that the designs obtained from both RTO approaches indeed have a lower standard deviation of the compliance than the deterministic design. The values estimated by the FOSM method deviate from the results obtained from Monte Carlo simulations with 1000 realizations, which is not surprising due to the assumption of linearity of the objective function implicitly made using the FOSM approach. The discrepancy however is much smaller than for the use case with random load. Though the FOSM approach underestimates the standard deviation significantly, the design obtained using FOSM in the RTO is more robust than the deterministically optimized design.

The SOFM method could not be used, because the required computational cost was too high for this case due to the model size.

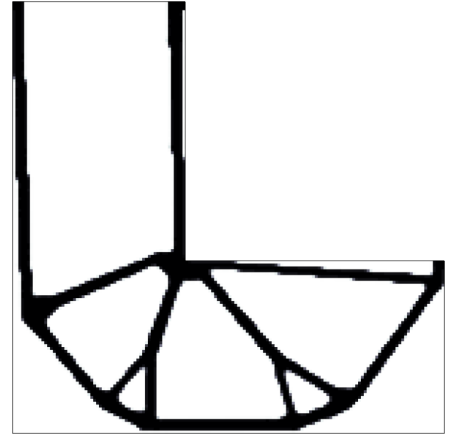

Fig. 17 Result of robust topology optimization of the L-beam with random geometry using FOSM

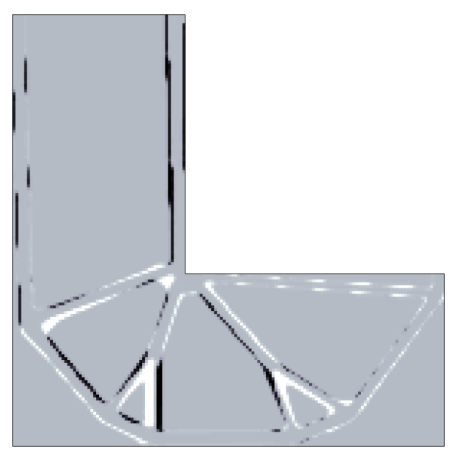

Fig. 19 Difference of deterministic and RTO results using FOSM, grey: equal, black: deterministic, white: FOSM

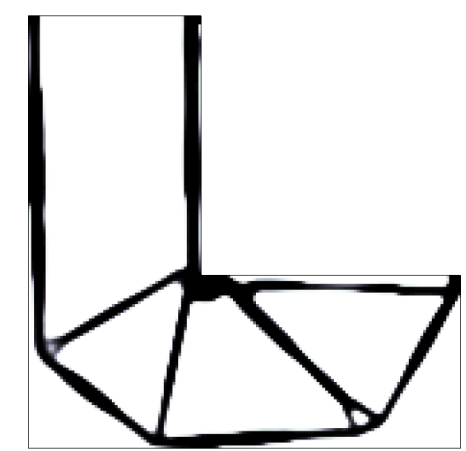

Fig. 18 Result of robust topology optimization of the L-beam with random geometry using Monte Carlo

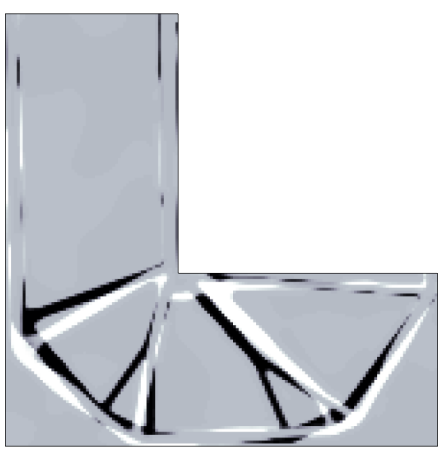

Fig. 20 Difference of deterministic and RTO results using Monte Carlo, grey: equal, black: deterministic, white: Monte Carlo

For the investigation of the influence of a spatial varying, random Young's modulus, a standard deviation of 0.1 (and hence, $10 \%$ of the mean) was chosen and the same correlation length as for the random geometry is used. The design obtained from RTO using FOSM and Monte Carlo hardly differ from the deterministic design. The same holds for the mean and standard deviation of the compliance, given in table 5. Optimizations with a much higher 
standard deviation of the Young's modulus provide designs that are more different. However, compared to most materials a standard deviation of $10 \%$ is already relatively large.

Table 5 Mean value and standard deviation of the compliance of the L-beam designs obtained by different RTO approaches, determined by Monte Carlo simulation with 1000 realizations

\begin{tabular}{lllll}
\hline \hline & \multicolumn{2}{c}{ Random geometry } & \multicolumn{2}{c}{ Random Young's modulus } \\
Approach & $\mu_{c}$ & $\sigma_{c}$ & $\mu_{c}$ & $\sigma_{c}$ \\
\hline Deterministic & 459 & 42.6 & 395.2 & 9.5 \\
Monte Carlo & 416 & 18.3 & 393.5 & 9.5 \\
FOSM & $438^{*}, 476$ & $10.6^{*}, 29.3$ & $389.3^{*}, 394.6$ & $9.4^{*}, 9.5$ \\
\hline \hline
\end{tabular}

\subsection{RTO of a cantilever beam random geometry}

In this section, the FOSM based approached is applied to the cantilever beam considered by Lazarov et al. [22], considering random geometry. The properties are summarized in table 6. Lazarov et al. used a PDE filter with a filter parameter of $R_{P D E}=0.01$. According to [23], this corresponds to a filter radius of $R=2 \sqrt{3} R_{P D E}=0.0346$ when using the density filter (17), which is used here. The correlation length of 2.5 is relatively large compared to the dimensions of the design space. This is beneficial when using the discrete KarhunenLoève transform 41, since "stochastic fields with large correlation lengths compared with the physical domain dimensions can be represented with a small number of the expansion modes." [22]

Table 6 Properties of the cantilever beam example, according to [22]

\begin{tabular}{ll}
\hline \hline Dimensions: & $4 \times 1$ \\
Element size & $L_{e}=0.01$ \\
Volume fraction & $V / V_{0}=50 \%$ \\
Filter radius & $r=0.0346$ \\
Applied load & $F=0.1$ \\
Correlation lengths & $l_{c}=2.5$ \\
\hline \hline
\end{tabular}

Since in [22] padding was not used at the design domain edges, results are determined with and without padding. The deterministically optimized design with and without padding are given in figure 21. Unsurprisingly, the when using padding and thereby considering geometric variability along the design space edge yields a higher standard deviation. This demonstrates the importance of using padding for the filter along the design domain edges when considering geometric variability.

For this example, the convergence behavior of the RTO using FOSM was very sensitive to the continuation of $\beta$. Here, $\beta$ was increased by 1.0 every 50 iterations. Still, the robustness optimizations diverged at some point. Therefore, the best results found during the optimization are given, which were obtained after 153 (with padding) and 151 (without padding) iterations. The obtained designs are shown on 22. For the case of no padding, the 
inaccuracy of the FOSM approach, caused by the assumption of linearity, provided a result, which is actually less robust than the deterministic design. For the case of using padding, the robustness was improved by the RTO. The optimal design found by [22] was not reached.
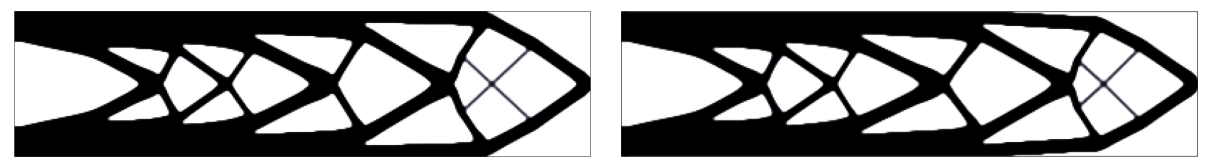

Fig. 21 Result of deterministic topology optimization without padding (left) and with padding (right) of the cantilever beam considered in [22]
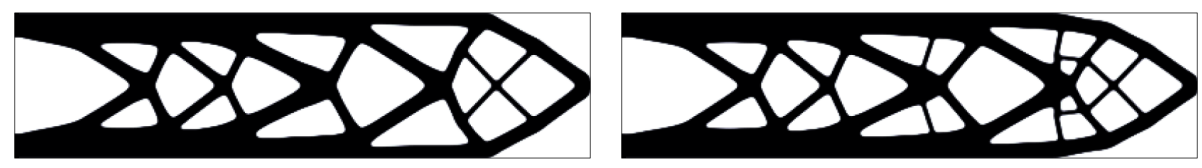

Fig. 22 Result of robust topology optimization using FOSM of the cantilever beam considered in [22] with random geometry without padding (left) and with padding (right)

Table 7 Mean value and standard deviation of the compliance of the cantilever beam, determined by Monte Carlo simulations with 1000 realizations

\begin{tabular}{lllll}
\hline \hline \multirow{2}{*}{ Approach } & $\mu_{c}$ & $\sigma_{c}$ & $\mu_{c}$ & with padding \\
\hline Deterministic & 3.66 & 0.138 & 3.74 & 0.211 \\
\hline FOSM & $3.83^{*}, 3.85$ & $0.090^{*}, 0.176$ & $4.10^{*}, 3.85$ & $0.165^{*}, 0.179$ \\
\hline $\begin{array}{l}\text { Second order } \\
\text { perturbation approach** }\end{array}$ & 3.68 & 0.083 & \\
\hline & $*$ determined by FOSM, **values from [22]
\end{tabular}

\subsection{RTO under distributed load with random geometry}

As a last example, a problem with a distributed load shown in figure 23 is considered, which is similar to an example in [37]. The dimensions and parameters are summarized in table 8. Due to the evenly distributed load, the deterministic optimization provides a design with gray elements (density between 0 and 1 ) in the area of the load introduction (see figure 24). Therefore, the design is very sensitive to variations in the threshold parameter $\eta$, which is why the interval of $\eta$ is chosen to be $[0.4,0.6]$. The effect can easily be avoided by adding a solid layer to the load introduction as non-design space. The example was nevertheless chosen as it is in order to shown the capabilities of the RTO approach in presence of significant sensitivity.

The designs obtained from deterministic and robustness optimization are shown in figure 24 and 25 . The design derived by the RTO is more solid in the load introduction area 


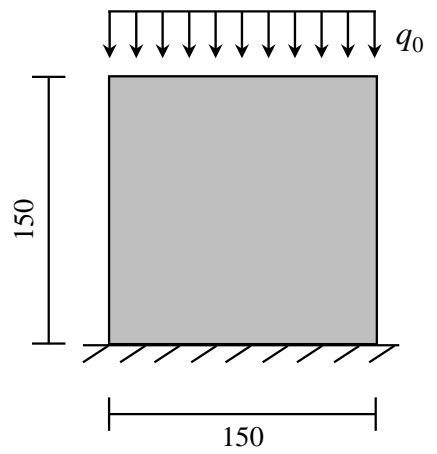

Fig. 23 Design space and load of an example with distributed load

Table 8 Properties of distributed load example, similar to [37]

\begin{tabular}{ll}
\hline \hline Dimensions: & $150 \times 150$ \\
Element size & $L_{e}=1$ \\
Volume fraction & $V / V_{0}=30 \%$ \\
Filter radius & $r=4$ \\
Applied load & $q_{0}=1$ \\
Correlation lengths & $l_{c}=20$ \\
\hline \hline
\end{tabular}

than the deterministically optimized design, which causes a reduction of sensitivity. The mean values and standard deviations of these design are determined by Monte Carlo simulations with 10000 realizations, and are given in table 9.

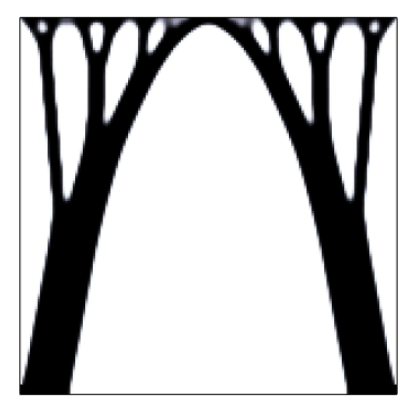

Fig. 24 Result of deterministic topology optimization of the distributed load example

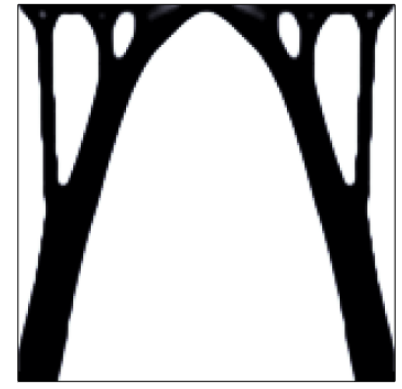

Fig. 25 Result of robust topology optimization of the distributed load example with random geometry using FOSM

\section{Conclusions and Outlook}

A robust topology optimization approach is presented, which utilizes the first-order secondmoment (FOSM) method for the estimation of mean value and variance of the compliance. The approach requires very little computational cost independently of the number of ran- 
Table 9 Mean value and standard deviation of the compliance of the distributed load example, determined by Monte Carlo simulations with 10000 realizations

\begin{tabular}{lll}
\hline \hline Approach & $\mu_{c}$ & $\sigma_{c}$ \\
\hline Deterministic & 984 & 20.9 \\
FOSM & $1036^{*}, 1043$ & $10.6^{*}, 11.8$ \\
\hline \hline \multicolumn{3}{c}{ *determined by FOSM $^{2}$}
\end{tabular}

dom parameters, since only one adjoint system needs to be solved per iteration. The FOSM approach lacks accuracy, because it is based on a first-order Taylor series expansion of the objective function. The objective function considered in this paper is the compliance of a structure and the random parameters are the applied load, the geometry or the spatially varying Young's modulus. The approach is applied to different use cases. For a random load, the FOSM approach is not applicable. For random geometry and Young's modulus however, the FOSM approach provides reasonable results at low computational cost, even though it does not estimate the variance of the compliance correctly. The importance of a correct filter function at the edges of the design domain especially for geometric deviations is demonstrated.

Future work should focus on RTO considering the stress, as this is an important optimization problem in practice and at the same time, the stress is expected to be more sensitive to local variations in geometry and material properties.

\section{A Appendix}

\section{A.1 Transformation to uncorrelated parameters}

Equations (7) and (8) are only valid for uncorrelated variables. (Eq. (8) actually furthermore requires independence for its derivation. Gaussian random variables are independent if they are uncorrelated, but that does not hold for all types of distributions.) Correlated variables therefore need to be transformed to uncorrelated variables.

A random vector $\mathbf{X}$ with mean vector $\boldsymbol{\mu}$ and covariance matrix $\boldsymbol{\Sigma}$ can be transformed to a random vector $\mathbf{Z}$ with uncorrelated entries by

$$
\mathbf{x}=\boldsymbol{\Sigma}^{\frac{1}{2}} \mathbf{z}+\boldsymbol{\mu}
$$

The mean values of the entries of $\mathbf{Z}$ equals 0 , the variances are normalized to 1 and all covariances of two entries equal 0. Note that if $\mathbf{X}$ follows gaussian distribution, all entries of $\mathbf{Z}$ are independent, i.e. all joint moments equal 0 . For other $\mathbf{Z}$ are still uncorrelated but not necessarily independent.

The derivative of eq. (35) equals

$$
\frac{\partial \mathbf{x}}{\partial \mathbf{z}}=\Sigma^{\frac{1}{2}}
$$

If the objective function $g$ is given as a function of correlated variables $\mathbf{X}$, the transformation is used to allow using the above given approaches for uncorrelated variables $\mathbf{Z}$. The derivatives of $g$ with respect to $\mathbf{x}$ are the transformed to the derivatives with respect to $\mathbf{z}$ by using the chain rule.

$$
\frac{\partial g}{\partial \mathbf{z}}=\left(\frac{\partial \mathbf{x}}{\partial \mathbf{z}}\right)^{T} \frac{\partial g}{\partial \mathbf{x}}=\left(\Sigma^{\frac{1}{2}}\right)^{T} \frac{\partial g}{\partial \mathbf{x}}
$$

Similarly, the mixed second partial derivative of $g$ equals

$$
\frac{\partial^{2} g}{\partial \mathbf{z} \partial y_{k}}=\left(\Sigma^{\frac{1}{2}}\right)^{T} \frac{\partial^{2} g}{\partial \mathbf{x} \partial y_{k}}
$$

and the third derivative reads

$$
\frac{\partial^{3} g}{\partial \mathbf{z} \partial \mathbf{z} \partial y_{k}}=\left(\Sigma^{\frac{1}{2}}\right)^{T} \frac{\partial^{3} g}{\partial \mathbf{x} \partial \mathbf{x} \partial y_{k}} \Sigma^{\frac{1}{2}}
$$


The root of the covariance matrix $\boldsymbol{\Sigma}$ can be found from the spectral decomposition of $\boldsymbol{\Sigma}$.

$$
\Sigma^{\frac{1}{2}}=\mathbf{Q} \mathbf{D}^{\frac{1}{2}}
$$

Here, $\mathbf{Q}$ is the matrix with eigenvectors $\mathbf{q}_{i}$ and $\mathbf{D}$ is a diagonal matrix with eigenvalues $\lambda_{i}$ of $\boldsymbol{\Sigma}$. Then, eq. (35) can also be written as

$$
\mathbf{x}=\boldsymbol{\mu}+\sum_{i=1}^{m} \mathbf{q}_{i} \sqrt{\lambda_{i}} z_{i}
$$

This representation shows that the transformation (35) is similar to principal component analysis or discrete Karhunen-Loève transform. It is often used to reduce the number of random variables, (i.e. the dimensionality of the design space), by choosing the number of modes considered $m$ to be smaller than the number of correlated random variables $n$ (i.e. the length of $\mathbf{x}$ ). The argument for neglecting an eigenvector is that the associated eigenvalue and hence, the variance in the direction of the eigenvector, is small. However, a small variance does not necessarily mean that the variation in this direction has a low impact on the response function. To illustrate that, consider a homogeneous 2D random field with exponential correlation function (42).

$$
C=\exp \left(-\frac{\left|x_{2}-x_{1}\right|^{2}}{l_{c}^{2}}\right)
$$

The random field has the dimension $40 \times 20$, it is discretized by $80 \times 40$ elements and the correlation length equals $l_{c}=4$. In figure 26 four eigenvectors (or eigenmodes) of the correlation matrix are shown. Modes 1 and 7 correspond to large variation, but describe only a gradually spatial variation. In contrast to that, modes 75 and 109 represent very short-range spatial variation, which is more critical for the applications considered in this paper (random Young's modulus and random geometry). For that reason, in the current paper the transformation (35) is only used to transform correlated to uncorrelated parameters, but not to reduce the number of parameters.
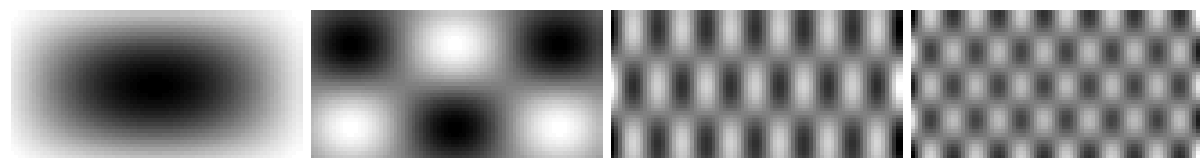

Fig. 26 Karhunen-Loève modes/eigenvectors 1, 7, 75 and 109 of an example random field

\section{A.2 Heaviside projection approximation and its derivatives}

At the end of the topology optimization using the SIMP approach, the filtered design variable $\tilde{\rho}$ is projected to a values of either 0 or 1 in order to avoid elements with intermediate density. This projection is done by the Heaviside function. In the current work follows the approach of performing the Heaviside projection in each iteration step, mainly because this allows modeling the random variation of the geometry as suggested by Schevenels et al. [29]. Since the Heaviside function is not differentiable, it is approximated by the projection function proposed by Wang et al. [34].

$$
\overline{\tilde{\rho}}=\frac{\tanh (\beta \eta)+\tanh (\beta(\tilde{\rho}-\eta))}{\tanh (\beta \eta)+\tanh (\beta(1-\eta))}
$$

For the mapping of the derivatives with respect to $\overline{\tilde{\rho}}$ derivatives with respect to $\rho$ as in eq. (49), the derivative of the projection function with respect to the filtered variable is required.

$$
\frac{\partial \overline{\tilde{\rho}}}{\partial \tilde{\rho}}=\frac{\beta(\operatorname{sech}(\beta(\tilde{\rho}-\eta)))^{2}}{\tanh (\beta \eta)+\tanh (\beta(1-\eta))}
$$

Furthermore, for the derivatives of the stiffness matrix with respect to $\eta$ in eq. (80), (82), (84) and (87), the first and second derivative of the projection function with respect to $\eta$ are required, which are given by

$$
\frac{\partial \overline{\tilde{\rho}}}{\partial \eta}=\beta \frac{u_{1}}{v}-\beta \frac{u_{2} u_{3}}{v}
$$


and

$$
\frac{\partial^{2} \overline{\tilde{\rho}}}{\partial \eta^{2}}=\beta \frac{u_{1}^{\prime} v-u_{1} v^{\prime}}{v^{2}}-\beta \frac{u_{2}^{\prime} u_{3} v+u_{2} u_{3}^{\prime} v-u_{2} u_{3} v^{\prime}}{v^{2}}
$$

with

$$
\begin{aligned}
u_{1} & =\tanh ^{2}(\beta(\tilde{\rho}-\eta))-\tanh ^{2}(\beta \eta) \\
u_{2} & =\tanh (\beta(\tilde{\rho}-\eta))+\tanh (\beta \eta) \\
u_{3} & =\tanh (\beta(1-\eta))-\tanh (\beta \eta) \\
v & =\tanh (\beta(1-\eta))+\tanh (\beta \eta) \\
u_{1}^{\prime} & =2 \beta\left[-\tanh (\beta(\tilde{\rho}-\eta))+\tanh ^{3}(\beta(\tilde{\rho}-\eta))-\tanh (\beta \eta)+\tanh ^{3}(\beta \eta)\right] \\
u_{3}^{\prime} & =\beta\left[\tanh ^{2}(\beta(1-\eta))+\tanh ^{2}(\beta \eta)-2\right] \\
u_{2}^{\prime} & =\beta\left[\tanh ^{2}(\beta(\tilde{\rho}-\eta))-\tanh ^{2}(\beta \eta)\right] \\
v^{\prime} & =\beta\left[\tanh ^{2}(\beta(1-\eta))-\tanh ^{2}(\beta \eta)\right]
\end{aligned}
$$

\section{A.3 Derivatives of compliance}

In the current paper, $\mu_{c}$ and $\sigma_{c}$ are estimated using the FOSM and SOFM approach. Hence, the derivatives summarized in eq. (13) (in terms of $y$ and $z$ ) are required to solve the optimization problem with a gradient based approach. In the following subsections, these derivatives are given for different random parameters, namely for load, Young's modulus and geometry. In any case, also the derivatives of compliance with respect to the design variables are required. The derivative of $c$ with respect to the projected variable $\tilde{\rho}$ is given by

$$
\frac{\partial c}{\partial \tilde{\tilde{\rho}}_{k}}=-\mathbf{u}^{T} \frac{\partial \mathbf{K}}{\partial \tilde{\tilde{\rho}}_{k}} \mathbf{u}
$$

Then, by application of the chain rule, the derivative with respect to the design variable $\rho$ is obtained.

$$
\frac{\partial c}{\partial \rho_{k}}=\frac{\partial c}{\partial \tilde{\tilde{\rho}}_{e}} \frac{\partial \overline{\tilde{\rho}}_{e}}{\partial \tilde{\rho}_{e}} \frac{\partial \tilde{\rho}_{e}}{\partial \rho_{k}}
$$

The derivative of the stiffness matrix can be determined on element level. Using the SIMP approach, the element stiffness matrix of the $i$-th element equals

$$
\mathbf{k}_{i}=\overline{\tilde{\rho}}^{p} \mathbf{k}_{0}
$$

and derivatives are given by

$$
\begin{aligned}
& \frac{\partial \mathbf{k}_{i}}{\partial \overline{\tilde{\rho}}_{k}}= \begin{cases}p \overline{\tilde{\rho}}_{i}^{p-1} \mathbf{k}_{0} & \text { for } i=k \\
0 & \text { else }\end{cases} \\
& \frac{\partial^{2} \mathbf{k}_{i}}{\partial \tilde{\tilde{\rho}}_{j} \partial \tilde{\tilde{\rho}}_{k}}= \begin{cases}p(p-1) \overline{\tilde{\rho}}_{i}^{p-2} \mathbf{k}_{0} & \text { for } i=j=k \\
0 & \text { else }\end{cases} \\
& \frac{\partial^{3} \mathbf{k}_{e}}{\partial \tilde{\tilde{\rho}}_{i} \partial \tilde{\tilde{\rho}}_{j} \partial \tilde{\tilde{\rho}}_{k}}= \begin{cases}p(p-1)(p-2) \tilde{\tilde{\rho}}_{i}^{p-3} \mathbf{k}_{0} & \text { for } e=i=j=k \\
0 & \text { else }\end{cases}
\end{aligned}
$$

In multiple derivatives shown in the subsequent section, the derivative of the displacement vector $\mathbf{u}$ with respect to projected variable $\overline{\tilde{\rho}}$ is required, which is given by

$$
\frac{\partial \mathbf{u}}{\partial \tilde{\tilde{\rho}}_{k}}=-\mathbf{K}^{-1} \frac{\partial \mathbf{K}}{\partial \overline{\tilde{\rho}}_{k}} \mathbf{u}
$$

Instead determining the inverse of $\mathbf{K}$, the following system is solved.

$$
\mathbf{K} \frac{\partial \mathbf{u}}{\partial \tilde{\tilde{\rho}}_{k}}=-\frac{\partial \mathbf{K}}{\partial \tilde{\tilde{\rho}}_{k}} \mathbf{u}
$$

Since the stiffness matrix $\mathbf{K}$ needs to be decomposed only once, the solution is relatively fast. Still, the number of times this system of linear equation needs to be solved is the main driver for the computational cost, if these entities are explicitly required. 


\section{A.3.1 Minimizing compliance with random load}

When considering entries of the load vector $f_{i}$ as random parameters, the following derivative of the compliance with respect to the loads needs to be determined.

$$
\frac{\partial c}{\partial f_{i}}, \frac{\partial c}{\partial \rho_{k}}, \frac{\partial^{2} c}{\partial f_{i} \partial f_{j}}, \frac{\partial^{2} c}{\partial f_{i} \partial \rho_{k}}, \frac{\partial^{3} c}{\partial f \partial f_{j} \partial \rho_{k}}
$$

The differentiation of $c$ w.r.t. $f$ reads

$$
\frac{\partial c}{\partial f_{i}}=\left(\frac{\partial \mathbf{u}}{\partial f_{i}}\right)^{T} \mathbf{f}+\mathbf{u}^{T} \frac{\partial \mathbf{f}}{\partial f_{i}}
$$

where

$$
\frac{\partial \mathbf{f}}{\partial f_{i}}=\mathbf{e}_{i}
$$

$\mathbf{e}_{i}$ is the unit vector. For determining $\frac{\partial \mathbf{u}}{\partial f_{i}}$ the equilibrium $(\mathbf{K u}=\mathbf{f})$ is differentiated w.r.t. $f_{i}$.

$$
\underbrace{\frac{\partial \mathbf{K}}{\partial f_{i}}}_{=0} \mathbf{u}+\mathbf{K} \frac{\partial \mathbf{u}}{\partial f_{i}}=\frac{\partial \mathbf{f}}{\partial f_{i}}=\mathbf{e}_{i} \Rightarrow \frac{\partial \mathbf{u}}{\partial f_{i}}=\mathbf{K}^{-1} \mathbf{e}_{i}
$$

Inserting this in (55) yields

$$
\frac{\partial c}{\partial f_{i}}=\left(\mathbf{K}^{-1} \mathbf{e}_{i}\right)^{T} \mathbf{f}+\mathbf{u}^{T} \mathbf{e}_{i}=\mathbf{e}_{i}^{T} \underbrace{\mathbf{K}^{-T} \mathbf{f}}_{\mathbf{u}}+\mathbf{u}^{T} \mathbf{e}_{i}=2 \mathbf{u}^{T} \mathbf{e}_{i}=2 u_{i}
$$

Hence, the first derivative of $c$ simply reads

$$
\frac{\partial c}{\partial f_{i}}=2 u_{i}
$$

Differentiating (59) with respect to $\overline{\tilde{\rho}}$ yields

$$
\frac{\partial^{2} c}{\partial f_{i} \partial \overline{\tilde{\rho}}_{k}}=2 \frac{\partial u_{i}}{\partial \tilde{\tilde{\rho}}_{k}}
$$

For SOFM, (59) needs to be differentiated with respect to another entry of the load vector $f_{j}$, which reads

$$
\frac{\partial^{2} c}{\partial f_{i} \partial f_{j}}=2 \frac{\partial u_{i}}{\partial f_{j}}=2 \bar{K}_{i j}
$$

where $\bar{K}_{i j}$ is the entry of the inverse stiffness matrix. In order to avoid determining the inverse of $\mathbf{K}, \frac{\partial u_{i}}{\partial f_{j}}$ can be determined by solving the following system (once per random entry of the load vector).

$$
\mathbf{K} \frac{\partial \mathbf{u}}{\partial f_{j}}=\mathbf{e}_{j}
$$

The SOFM method furthermore requires the third derivative of the compliance. For that, eq. (60) is written as

$$
\frac{\partial^{2} c}{\partial f_{i} \partial \overline{\tilde{\rho}}_{k}}=2 \frac{\partial u_{i}}{\partial \overline{\tilde{\rho}}_{k}}=2 \mathbf{e}_{i}^{T}\left(-\mathbf{K}^{-1} \frac{\partial \mathbf{K}}{\partial \overline{\tilde{\rho}} k} \mathbf{u}\right)
$$

Differentiating this expression w.r.t. $f_{j}$ provides

$$
\frac{\partial^{3} c}{\partial f_{i} \partial f_{j} \partial \overline{\tilde{\rho}}_{k}}=-2 \mathbf{e}_{i}^{T} \mathbf{K}^{-1} \frac{\partial \mathbf{K}}{\partial \overline{\tilde{\rho}}_{k}} \frac{\partial \mathbf{u}}{\partial f_{j}}
$$

In order to avoid determining the inverse, the derivatives can be determined by solving

$$
\mathbf{K} \frac{\partial^{3} c}{\partial \mathbf{f} \partial f_{j} \partial \overline{\tilde{\rho}}_{k}}=-2 \frac{\partial \mathbf{K}}{\partial \overline{\tilde{\rho}}_{k}} \frac{\partial \mathbf{u}}{\partial f_{j}}
$$

The derivatives (60) and (64) are given with respect to the projected variable. The derivatives with respect to the design variable is obtained by applying the chain rule as in eq. (49).

Given a finite element model with $n$ elements and $m$ random loads, $n$ systems need to be solved for eq. (60), $m$ systems need to be solved for eq. (61), and the system (65) needs to be solved $n \times m$ times. 


\section{A.3.2 Minimizing compliance with random Young's modulus}

Next, the Young's modulus is considered as a spatially varying parameter. Hence, the Young's moduli $E_{i}$ of each finite element are considered as correlated random variables. The derivatives that need to be determined are

$$
\frac{\partial c}{\partial E_{i}}, \frac{\partial c}{\partial \rho_{k}}, \frac{\partial^{2} c}{\partial E_{i} \partial \rho_{k}}, \frac{\partial^{2} c}{\partial E_{i} \partial E_{j}}, \frac{\partial^{3} c}{\partial E_{i} E_{j} \partial \rho_{k}}
$$

The derivative of $c$ w.r.t. the Young's modulus reads

$$
\frac{\partial c}{\partial E_{i}}=\mathbf{f}^{T} \frac{\partial \mathbf{u}}{\partial E_{i}}+\mathbf{u}^{T} \underbrace{\frac{\partial \mathbf{f}}{\partial E_{i}}}_{=0}
$$

Differentiating the equilibrium system $(\mathbf{K} \mathbf{u}=\mathbf{f})$ w.r.t. $E_{i}$ reads

$$
\frac{\partial \mathbf{K}}{\partial E_{i}} \mathbf{u}+\mathbf{K} \frac{\partial \mathbf{u}}{\partial E_{i}}=\underbrace{\frac{\partial \mathbf{f}}{\partial E_{i}}}_{=0} \Rightarrow \frac{\partial \mathbf{u}}{\partial E_{i}}=-\mathbf{K}^{-1} \frac{\partial \mathbf{K}}{\partial E_{i}} \mathbf{u}
$$

Inserting this into eq. (67) yields

$$
\frac{\partial c}{\partial E_{i}}=\mathbf{f}^{T}\left(-\mathbf{K}^{-1} \frac{\partial \mathbf{K}}{\partial E_{i}} \mathbf{u}\right)=-\mathbf{u}^{T} \frac{\partial \mathbf{K}}{\partial E_{i}} \mathbf{u}
$$

The derivative of the stiffness matrix $\mathbf{K}$ with respect to $E_{i}$ is obtained from the element stiffness matrix $\mathbf{k}_{\mathbf{i}}$ of the $i$-th element. The element stiffness matrix is given by

$$
\mathbf{k}_{i}\left(E_{i}, \rho_{i}\right)=\frac{E_{i}}{E_{0}} \overline{\tilde{\rho}}_{i}^{p} \mathbf{k}_{0}
$$

where $\mathbf{k}_{\mathbf{0}}$ is the unscaled element stiffness matrix with the initial Young's modulus $E_{0}$. Hence, the derivative equals

$$
\frac{\partial \mathbf{k}_{i}}{\partial E_{j}}=\left\{\begin{array}{lr}
\frac{1}{E_{0}} \overline{\tilde{\rho}}_{i}^{p} \mathbf{k}_{0} & \text { for } i=j \\
0 & \text { else }
\end{array}\right.
$$

and

$$
\frac{\partial^{2} \mathbf{k}_{i}}{\partial E_{j} \partial \overline{\tilde{\rho}}_{k}}=\left\{\begin{array}{lr}
\frac{1}{E_{0}} p \overline{\tilde{\rho}}_{i}^{p-1} \mathbf{k}_{0} & \text { for } i=j=k \\
0 & \text { else }
\end{array}\right.
$$

The second derivative of $\mathbf{k}_{i}$ with respect to $E_{i}$ equals zero.

The second derivatives of the compliance are given by

$$
\frac{\partial^{2} c}{\partial E_{i} \partial \overline{\tilde{\rho}}_{j}}=-2 \mathbf{u}^{T} \frac{\partial \mathbf{K}}{\partial E_{i}} \frac{\partial \mathbf{u}}{\partial \tilde{\tilde{\rho}}_{j}}-\mathbf{u}^{T} \frac{\partial \mathbf{K}}{\partial E_{i} \partial \overline{\tilde{\rho}}_{j}} \mathbf{u}
$$

and

$$
\frac{\partial^{2} c}{\partial E_{i} \partial E_{j}}=-2 \mathbf{u}^{T} \frac{\partial \mathbf{K}}{\partial E_{i}} \frac{\partial \mathbf{u}}{\partial E_{j}}
$$

The derivative of $\mathbf{u}$ with respect to $E_{i}$ is determined by solving

$$
\mathbf{K} \frac{\partial \mathbf{u}}{\partial E_{j}}=-\frac{\partial \mathbf{K}}{\partial E_{j}} \mathbf{u}
$$

The third derivative of the compliance reads

$$
\frac{\partial^{3} c}{\partial E_{i} \partial E_{j} \partial \overline{\tilde{\rho}}_{k}}=-2\left(\frac{\partial \mathbf{u}}{\partial \overline{\tilde{\rho}}_{k}}\right)^{T} \frac{\partial \mathbf{K}}{\partial E_{i}} \frac{\partial \mathbf{u}}{\partial E_{j}}-2 \mathbf{u}^{T} \frac{\partial \mathbf{K}}{\partial E_{i} \partial \overline{\tilde{\rho}}_{k}} \frac{\partial \mathbf{u}}{\partial E_{j}}-2 \mathbf{u}^{T} \frac{\partial \mathbf{K}}{\partial E_{i}} \frac{\partial \mathbf{u}}{\partial E_{j} \partial \overline{\tilde{\rho}}_{k}}
$$


For the second derivative of the displacement vector, the following system of linear equation is solved.

$$
\mathbf{K} \frac{\partial^{2} \mathbf{u}}{\partial E_{i} \partial \overline{\tilde{\rho}}_{k}}=-\frac{\partial \mathbf{K}}{\partial E_{i}} \frac{\partial \mathbf{u}}{\partial \overline{\tilde{\rho}}_{k}}-\frac{\partial^{2} \mathbf{K}}{\partial E_{i} \partial \overline{\tilde{\rho}}_{k}} \mathbf{u}-\frac{\partial \mathbf{K}}{\partial \overline{\tilde{\rho}}_{k}} \frac{\partial \mathbf{u}}{\partial E_{i}}
$$

For eq. (73) the system (53) needs to be solved $n$ times. The derivatives (74) and (76) are not required for FOSM, but only for the SOFM approach. For the latter, eq. (75) needs to be solved $n$ times and eq. (77) needs to be solved $n^{2}$ times. In total, the SOFM approach required $2 n+n^{2}$ solutions of systems of linear equations.

Again, the derivatives are given with respect to the projected variable and need to be transformed to derivatives with respect to design variables as in eq. (49).

\section{A.3.3 Minimizing compliance with random geometry}

The randomness of the geometry is modeled as first proposed by Schevenels et al. [29]. The basic idea is to consider the projection threshold $\eta$ of the projection function (43) as a spatially varying random parameter. Hence, there is a different (but correlated) projection threshold $\eta_{i}$ for each finite element of the design space, which the following derivatives need to be derived.

$$
\frac{\partial c}{\partial \eta_{i}}, \frac{\partial c}{\partial \rho_{k}}, \frac{\partial^{2} c}{\partial \eta_{i} \partial \rho_{k}}, \frac{\partial^{2} c}{\partial \eta_{i} \partial \eta_{j}}, \frac{\partial^{3} c}{\partial \eta_{i} \partial \eta_{j} \partial \rho_{k}}
$$

The derivative of the compliance with respect to $\eta_{i}$ is given by

$$
\frac{\partial c}{\partial \eta_{i}}=-\mathbf{u}^{T} \frac{\partial \mathbf{K}}{\partial \eta_{i}} \mathbf{u}
$$

The derivation is similar to the derivation of (69).

The derivative of the stiffness matrix $\mathbf{K}$ with respect to the threshold $\eta$ is determined using the chain rule. The derivative of $\mathbf{K}$ with respect to the projected design parameter $\overline{\tilde{\rho}}$ is known (see eq. (51)) and the derivative of $\overline{\tilde{\rho}}$ with respect to $\eta$ is obtained from the chosen projection function, see eq. (45).

$$
\frac{\partial \mathbf{K}}{\partial \eta_{i}}=\frac{\partial \mathbf{K}}{\partial \tilde{\tilde{\rho}}_{i}} \frac{\partial \tilde{\tilde{\rho}}_{i}}{\partial \eta_{i}}
$$

The second derivatives with respect to $\eta$ and $\overline{\tilde{\rho}}$ reads

$$
\frac{\partial^{2} c}{\partial \eta_{i} \partial \tilde{\tilde{\rho}}_{k}}=-2 \mathbf{u}^{T} \frac{\partial \mathbf{K}}{\partial \eta_{i}} \frac{\partial \mathbf{u}}{\partial \tilde{\tilde{\rho}}_{k}}-\mathbf{u}^{T} \frac{\partial^{2} \mathbf{K}}{\partial \eta_{i} \partial \overline{\tilde{\rho}}_{k}} \mathbf{u}
$$

The derivatives of the first term are known. The second derivative of the $\mathbf{K}$ with respect to $\eta$ and $\overline{\tilde{\rho}}$ reads

$$
\frac{\partial^{2} \mathbf{K}}{\partial \eta_{i} \partial \tilde{\tilde{\rho}}_{k}}=\left\{\begin{array}{lr}
\frac{\partial^{2} \mathbf{K}}{\partial \overline{\tilde{\rho}}_{i} \partial \overline{\tilde{\rho}}_{i}} \frac{\partial \overline{\tilde{\rho}}_{i}}{\partial \eta_{i}} & \text { for } i=k \\
0 & \text { else }
\end{array}\right.
$$

The second derivatives with respect to different $\eta_{i}$ and $\eta_{j}$ equals

$$
\frac{\partial^{2} c}{\partial \eta_{i} \partial \eta_{j}}=-2 \mathbf{u}^{T} \frac{\partial \mathbf{K}}{\partial \eta_{i}} \frac{\partial \mathbf{u}}{\partial \eta_{j}}-\mathbf{u}^{T} \frac{\partial^{2} \mathbf{K}}{\partial \eta_{i} \partial \eta_{j}} \mathbf{u}
$$

Here, the second derivative of $\mathbf{K}$ with respect to different $\eta_{i}$ and $\eta_{j}$ is required, which is given by

$$
\frac{\partial^{2} \mathbf{K}}{\partial \eta_{i} \partial \eta_{j}}=\left\{\begin{array}{lr}
\frac{\partial^{2} \mathbf{K}}{\partial \tilde{\tilde{\rho}}_{i}^{2}}\left(\frac{\partial \overline{\tilde{\rho}}_{i}}{\partial \eta_{i}}\right)^{2}+\frac{\partial \mathbf{K}}{\partial \overline{\tilde{\rho}}_{i}} \frac{\partial^{2} \overline{\tilde{\rho}}_{i}}{\partial \eta_{i}^{2}} & \text { for } i=j \\
0 & \text { else }
\end{array}\right.
$$

Furthermore, the derivative of $\mathbf{u}$ with respect to $\eta$ is required. Similarly as eq. (53) it is determined from

$$
\mathbf{K} \frac{\partial \mathbf{u}}{\partial \eta_{j}}=-\frac{\partial \mathbf{K}}{\partial \eta_{j}} \mathbf{u}
$$


Finally, the third derivative of the compliance is given by

$$
\begin{aligned}
\frac{\partial^{3} c}{\partial \eta_{i} \partial \eta_{j} \partial \overline{\tilde{\rho}}_{k}}=-2\left(\frac{\partial \mathbf{u}}{\partial \overline{\tilde{\rho}}_{k}}\right)^{T} \frac{\partial \mathbf{K}}{\partial \eta_{i}} \frac{\partial \mathbf{u}}{\partial \eta_{j}}-2 \mathbf{u}^{T} & \underbrace{\frac{\partial^{2} \mathbf{K}}{\partial \eta_{i} \partial \overline{\tilde{\rho}}_{k}}}_{=0 \text { for } i \neq k} \frac{\partial \mathbf{u}}{\partial \eta_{j}}-2 \mathbf{u}^{T} \frac{\partial \mathbf{K}}{\partial \eta_{i}} \frac{\partial^{2} \mathbf{u}}{\partial \overline{\tilde{\rho}}_{j} \partial \overline{\tilde{\rho}}_{k}} \frac{\partial \overline{\tilde{\rho}}_{j}}{\partial \eta_{j}} \\
& -2 \mathbf{u}^{T} \underbrace{\frac{\partial^{2} \mathbf{K}}{\partial \eta_{i} \partial \eta_{j}}}_{=0 \text { for } i \neq k} \frac{\partial \mathbf{u}}{\partial \tilde{\tilde{\rho}}_{k}}-\mathbf{u}^{T} \underbrace{\frac{\partial^{3} \mathbf{K}}{\partial \eta_{i} \partial \eta_{j} \partial \tilde{\tilde{\rho}}_{k}}}_{=0 \text { for } i \neq j \neq k} \mathbf{u}
\end{aligned}
$$

Here, the third derivative of $\mathbf{K}$ is required, which is given by

$$
\frac{\partial^{3} \mathbf{K}}{\partial \eta_{i} \partial \eta_{j} \partial \overline{\tilde{\rho}}_{k}}=\left\{\begin{array}{lr}
\frac{\partial^{3} \mathbf{K}}{\partial \overline{\tilde{\rho}}_{i}^{3}}\left(\frac{\partial \overline{\tilde{\rho}}_{i}}{\partial \eta_{i}}\right)^{2}+\frac{\partial^{2} \mathbf{K}}{\partial \overline{\tilde{\rho}}_{i}^{2}} \frac{\partial^{2} \overline{\tilde{\rho}}_{i}}{\partial \eta_{i}^{2}} & \text { for } i=j=k \\
0 & \text { else }
\end{array}\right.
$$

The second derivative of $\mathbf{u}$ with respect to $\overline{\tilde{\rho}}_{i}$ is obtained by solving

$$
\mathbf{K} \frac{\partial^{2} \mathbf{u}}{\partial \tilde{\tilde{\rho}}_{j} \partial \tilde{\tilde{\rho}}_{k}}=-\underbrace{\frac{\partial^{2} \mathbf{K}}{\partial \tilde{\tilde{\rho}}_{j} \partial \overline{\tilde{\rho}}_{k}}}_{=0 \text { for } j \neq k} \mathbf{u}-\frac{\partial \mathbf{K}}{\partial \overline{\tilde{\rho}}_{k}} \frac{\partial \mathbf{u}}{\partial \tilde{\tilde{\rho}}_{j}}-\frac{\partial \mathbf{K}}{\partial \tilde{\tilde{\rho}}_{j}} \frac{\partial \mathbf{u}}{\partial \tilde{\tilde{\rho}}_{k}}
$$

For the FOSM method, the derivatives (79) and (81) are required, which involves solving (53) $n$ times. The SOFM approach additionally requires $n$ times solving (85) and $n^{2}$ times solving (88).

\section{References}

1. Asadpoure, A., Tootkaboni, M., Guest, J.K.: Robust topology optimization of structures with uncertainties in stiffness Application to truss structures. Computers \& Structures 89(11), 1131-1141 (2011). DOI 10.1016/j.compstruc.2010.11.004.

2. Bae, K.r., Wang, S., Choi, K.K.: Reliability-based topology optimization. In: 9th AIAA/ISSMO Symposium on Multidisciplinary Analysis and Optimization, pp. AIAA 2002-5542. Atlanta, Georgia, USA (2002)

3. Bendse, M.P.: Optimal shape design as a material distribution problem. Structural optimization 1(4), 193-202 (1989). DOI 10.1007/BF01650949.

4. Carrasco, M., Ivorra, B., Ramos, A.M.: Stochastic topology design optimization for continuous elastic materials. Computer Methods in Applied Mechanics and Engineering 289, 131-154 (2015). DOI 10.1016/j.cma.2015.02.003.

5. Clausen, A., Andreassen, E.: On filter boundary conditions in topology optimization. Structural and Multidisciplinary Optimization 56(5), 1147-1155 (2017). DOI 10.1007/s00158-017-1709-1.

6. Cornell, C.A.: Structural Safety Specifications Based on Second-Moment Reliability Analysis. IABSE reports of the working commissions 4, 235-245 (1969). DOI 10.5169/seals-5948.

7. Dunning, P.D., Kim, H.A., Mullineux, G.: Introducing Loading Uncertainty in Topology Optimization. AIAA Journal 49(4), 760-768 (2011). DOI 10.2514/1.J050670.

8. Elishakof, I.: Probabilistic Theory of Structures. Dover, Mineola, NY (1999)

9. Elishakoff, I.: An Idea of the Uncertainty Triangle. The Shock and Vibration Digest 22, 1 (1990)

10. de Gournay, F., Allaire, G., Jouve, F.: Shape and topology optimization of the robust compliance via the level set method. ESAIM: Control, Optimisation and Calculus of Variations 14(01), 43-70 (2008). DOI 10.1051/cocv:2007048.

11. Guo, X., Zhang, W., Zhang, L.: Robust structural topology optimization considering boundary uncertainties. Computer Methods in Applied Mechanics and Engineering 253, 356-368 (2013). DOI 10.1016/j.cma.2012.09.005.

12. Haldar, A., Mahadevan, S.: Probability, Reliability and Statistical Methods in Engineering Design, 1. auflage edn. John Wiley \& Sons, New York; Chichester England (1999).

13. Harzheim, L.: Strukturoptimierung: Grundlagen und Anwendungen. Harri Deutsch Verlag (2008).

14. Hasofer, A.M., Lind, N.C.: Exact and Invariant Second-Moment Code Format. Journal of the Engineering Mechanics Division pp. 111-121 (1974). 
15. Jalalpour, M., Guest, J.K., Igusa, T.: Reliability-based topology optimization of trusses with stochastic stiffness. Structural Safety 43, 41-49 (2013). DOI 10.1016/j.strusafe.2013.02.003.

16. Jalalpour, M., Tootkaboni, M.: An efficient approach to reliability-based topology optimization for continua under material uncertainty. Structural and Multidisciplinary Optimization 53(4), 759-772 (2016). DOI 10.1007/s00158-015-1360-7.

17. Jung, H.S., Cho, S.: Reliability-based topology optimization of geometrically nonlinear structures with loading and material uncertainties. Finite Elements in Analysis and Design 41(3), 311-331 (2004). DOI 10.1016/j.finel.2004.06.002.

18. Kharmanda, G., Olhoff, N., Mohamed, A., Lemaire, M.: Reliability-based topology optimization. Structural and Multidisciplinary Optimization 26(5), 295-307 (2004). DOI 10.1007/s00158-003-0322-7.

19. Kim, S.R., Park, J.Y., Lee, W.G., Yu, J.S., Han, S.Y.: Reliability-based topology optimization based on evolutionary structural optimization. Int J Mech Syst Sci Eng 1(3), 135-139 (2007).

20. Kriegesmann, B., Rolfes, R., Jansen, E.L., Elishakoff, I., Hhne, C., Kling, A.: Design Optimization of Composite Cylindrical Shells under Uncertainty. Computers, Materials, \& Continua 32(3), 177-200 (2012). DOI 10.3970/cmc.2012.032.177.

21. Lazarov, B.S., Schevenels, M., Sigmund, O.: Topology optimization considering material and geometric uncertainties using stochastic collocation methods. Structural and Multidisciplinary Optimization 46(4), 597-612 (2012). DOI 10.1007/s00158-012-0791-7.

22. Lazarov, B.S., Schevenels, M., Sigmund, O.: Topology optimization with geometric uncertainties by perturbation techniques. International Journal for Numerical Methods in Engineering 90(11), 13211336 (2012). DOI 10.1002/nme.3361.

23. Lazarov, B.S., Sigmund, O.: Filters in topology optimization based on Helmholtz-type differential equations. International Journal for Numerical Methods in Engineering 86(6), 765-781 (2011). DOI 10.1002/nme.3072.

24. Li, L., Khandelwal, K.: Two-point gradient-based MMA (TGMMA) algorithm for topology optimization. Computers \& Structures 131, 34-45 (2014). DOI 10.1016/j.compstruc.2013.10.010.

25. Lpez, C., Baldomir, A., Hernndez, S.: The relevance of reliability-based topology optimization in early design stages of aircraft structures. Structural and Multidisciplinary Optimization (2017). DOI $10.1007 / \mathrm{s} 00158-017-1740-2$

26. Luo, Y., Zhou, M., Wang, M.Y., Deng, Z.: Reliability based topology optimization for continuum structures with local failure constraints. Computers \& Structures 143, 73-84 (2014). DOI 10.1016/j.compstruc.2014.07.009

27. Maute, K., Frangopol, D.M.: Reliability-based design of MEMS mechanisms by topology optimization. Computers \& Structures 81(8), 813-824 (2003). DOI 10.1016/S0045-7949(03)00008-7.

28. Papadimitriou, D.I., Mourelatos, Z.P.: Reliability-Based Topology Optimization Using Mean-Value Second-Order Saddlepoint Approximation. Journal of Mechanical Design 140(3), 031,403-031,40311 (2018). DOI 10.1115/1.4038645.

29. Schevenels, M., Lazarov, B.S., Sigmund, O.: Robust topology optimization accounting for spatially varying manufacturing errors. Computer Methods in Applied Mechanics and Engineering 200(4952), 36133627 (2011). DOI 10.1016/j.cma.2011.08.006.

30. Schuller, G.I., Valdebenito, M.: Reliability-Based Optimization - An Overview. Computational Technology Reviews 1, 121-155 (2010)

31. Sigmund, O.: Manufacturing tolerant topology optimization. Acta Mechanica Sinica 25(2), 227-239 (2009). DOI 10.1007/s10409-009-0240-z.

32. Svanberg, K.: The method of moving asymptotesa new method for structural optimization. International Journal for Numerical Methods in Engineering 24(2), 359-373 (1987). DOI 10.1002/nme.1620240207.

33. Torii, A., Novotny, A., dos Santos, R. B.: Robust Compliance Topology Optimization Based on the Topological Derivative Concept. International Journal for Numerical Methods in Engineering 106(11), 889-903 (2016). DOI https://doi.org/10.1002/nme.5144

34. Wang, F., Lazarov, B.S., Sigmund, O.: On projection methods, convergence and robust formulations in topology optimization. Structural and Multidisciplinary Optimization 43(6), 767-784 (2011). DOI $10.1007 / \mathrm{s} 00158-010-0602-\mathrm{y}$.

35. Yao, W., Chen, X., Luo, W., van Tooren, M., Guo, J.: Review of uncertainty-based multidisciplinary design optimization methods for aerospace vehicles. Progress in Aerospace Sciences 47(6), 450-479 (2011). DOI 10.1016/j.paerosci.2011.05.001.

36. Zang, T.A., Hemsch, M.J., Hilburger, M.W., Kenny, S.P., Luckring, J.M., Maghami, P., Padula, S.L., Stroud, W.J.: Needs and opportunities for uncertainty-based multidisciplinary design methods for aerospace vehicles. Tech. Rep. NASA/TM-2002-211462, National Aeronautics and Space Administration, Langley Research Center (2002)

37. Zhao, J., Wang, C.: Robust topology optimization under loading uncertainty based on linear elastic theory and orthogonal diagonalization of symmetric matrices. Computer Methods in Applied Mechanics and Engineering 273, 204-218 (2014). DOI 10.1016/j.cma.2014.01.018. 